\title{
Estimation of the microwave load parameters' measurement error, caused by imperfection of the probe, used for study of the electromagnetic wave's phase distribution in the transmission line
}

\author{
Yu.B. Gimpilevich and S.E. Zebek ${ }^{*}$ \\ SevSU Engineering Center Ltd, Sevastopol State University, Sevastopol, Russia
}

\begin{abstract}
The study concerns the influence of a non-ideal probe on a measurement error of the complex parameters of microwave nodes in the implementation of the quadrature measurement method based on the analysis of the electromagnetic wave's phase distribution in the transmission line. A mathematical model of the quadrature measurement method has been developed using an imperfect probe, and relations have been obtained that allow one to calculate measurement errors. A program for error modelling and calculating was developed and multiple numerical studies were performed using this program. It is shown that for practically important cases, the relative error in the measurement of the module does not exceed $0.5 \%$, and the absolute error of the argument does not exceed 2 degrees.
\end{abstract}

\section{Introduction}

Measurement of the complex parameters of microwave nodes allows you to get the most complete information about the properties of these nodes. In microwave technology, vector meters are widely used for these purposes, which make it possible to determine both the modules and the arguments of complex parameters [1-5]. One of the widely used methods for measuring the complex parameters of microwave loads is the measuring line method $[1,2]$, which provides the measurement of complex parameters both in manual mode using a probe moved along the transmission line [1,2], and in automatic mode using several motionless probes $[3,4]$. In the classical form, this method is implemented based on the analysis of the amplitude distribution of the electromagnetic field in the transmission line by amplitude quadratic detection of branched microwave oscillations [1-4]. The disadvantage of this measurement method is the presence of an error due to the nonsquareness of the voltampere characteristics of the microwave diodes [3,4]. The dynamic range of acceptable quadratic detection for these diodes is small and is $(30-40 \mathrm{~dB})[3,4]$. This circumstance leads to a significant error in the measurement of large values of the reflection coefficient, as well as the inability to carry out measurements under the conditions of the built-in control, under which the power of the microwave generator can vary over wide limits. A

\footnotetext{
* Corresponding author: stanislavzebek@mail.ru
} 
significant increase in the dynamic range of the input signal to (70-80) $\mathrm{dB}$ was achieved in the works of the authors of this article due to the transition to the analysis of the phase distribution of the field in the transmission line [6-9]. In [6], a phasometric measurement method was developed using only the phase distribution of the electromagnetic field in the transmission line, and in [7-9], a quadrature measurement method was developed using both the amplitude and phase distribution of the field in the transmission line. One of the sources of measurement error when using probe structures is the non-ideal characterization of the branch probe. A study of the influence of a non-ideal probe on measurement errors when using the analysis of the amplitude distribution of the electromagnetic field in the transmission line was carried out in a number of works, for example, in [10]. At the same time, similar studies using the analysis of the phase distribution of the electromagnetic field in the transmission line have not yet been carried out.

The aim of the work is to evaluate the errors in the measurement of the module and the argument of the complex reflection coefficient (CRC) of a microwave two-terminal network caused by the imperfection of an omnidirectional probe when implementing the quadrature measurement method using analysis of the phase distribution of the electromagnetic wave in the transmission line.

\section{Development of a mathematical model of a quadrature measurement method for the case of a non-ideal probe}

The microwave part of the circuit of the CRC meter implementing the quadrature method $[7,8]$ is shown in Fig. 1.

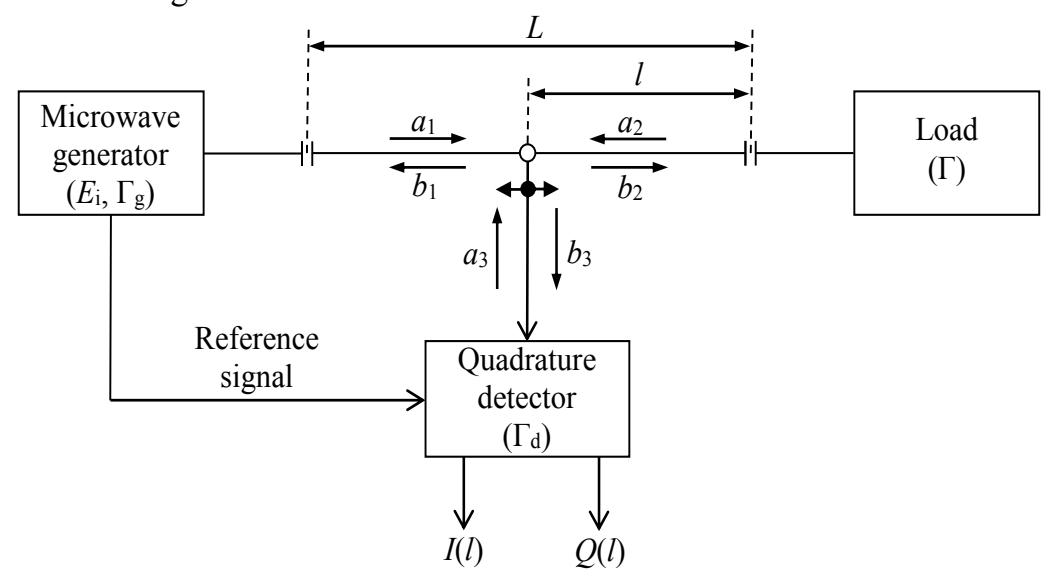

Fig. 1. Block diagram of the microwave part of the CRC meter circuit.

Harmonic oscillation from the microwave generator, through a length $L$ of the transmission line, is supplied to the final load, the CRC of which must be determined. An omnidirectional probe is introduced into this segment through a longitudinal slot $l$ at a certain distance from the load connection plane. The probe can move along a segment of the transmission line, and the distance $l$ will vary. The microwave signal branched by the probe is fed to the input of the quadrature detector, the reference input of which receives the reference oscillation, which is formed from the oscillation of the microwave generator, for example, using a directional coupler or a power divider. At the same time, the so-called quadrature components $I(l)$ and $Q(l)$ are formed at the outputs of the quadrature detector and, knowledge of which allows us to determine the initial phase of the branch signal when the probe is moving, that is, to restore the phase distribution of the electromagnetic wave 
along the transmission line. This, in turn, allows us to calculate the modulus and argument of the complex reflection coefficient of the final load using the formulas given in $[7,8]$.

In Fig. 1 is indicated: $E_{\mathrm{i}}$ — the amplitude of the incident wave excited in a segment of the transmission line in the plane of the generator connection (the initial phase is assumed to be zero); $\Gamma_{\mathrm{g}}-\mathrm{CRC}$ of microwave generator in the plane of its connection; $\Gamma-\mathrm{CRC}$ of load in the plane of its connection; $\Gamma_{d}-\mathrm{CRC}$ of the quadrature detector.

Upon further consideration, the probe will be considered imperfect and represent it in the form of a six-terminal, which is described by the scattering matrix $[S]$. We write the system of equations connecting the incident $a_{k}$ and reflected $b_{k}$ waves at the poles of this six-terminal network in the plane of connection of the probe [10]:

$$
\left\{\begin{array}{l}
b_{1}=S_{11} a_{1}+S_{12} a_{2}+S_{13} a_{3} \\
b_{2}=S_{21} a_{1}+S_{22} a_{2}+S_{23} a_{3} \\
b_{3}=S_{31} a_{1}+S_{32} a_{2}+S_{33} a_{3}
\end{array}\right.
$$

where $S_{n m}$ - are the elements of the six-terminal scattering matrix.

Let's write the boundary conditions at the poles of the six-port blocks:

$$
\left\{\begin{array}{l}
a_{1}=E_{\mathrm{i}} E_{\mathrm{i}} e^{-j \beta(L-l)}+b_{1} \Gamma_{\mathrm{r}} E_{\mathrm{i}} e^{-j \beta(L-l)} \\
a_{2}=b_{2} \Gamma e^{-j 2 \beta l} \\
a_{3}=b_{3} \Gamma_{\mathrm{d}}
\end{array}\right.
$$

$\beta=2 \pi / \lambda$ - phase constant; $\lambda$ - transmission line wavelength.

Substituting the boundary conditions (2) into the system of equations (1), taking into account that the six-terminal is mutual $\left(S_{n m}=S_{m n}\right)$ and has symmetry with respect to the plane of the probe ( $S_{11}=S_{22}, S_{31}=S_{32}$ ), we transform this system to the following form:

$$
\left\{\begin{array}{l}
-S_{11} E_{\mathrm{i}} e^{-j \beta(L-l)}=b_{1}\left(S_{11} \Gamma_{\mathrm{g}} e^{-j 2 \beta(L-l)}-1\right)+b_{2} S_{21} \Gamma e^{-j 2 \beta l}+b_{3} S_{31} \Gamma_{\mathrm{d}} \\
-S_{21} E_{\mathrm{i}} e^{-j \beta(L-l)}=b_{1} S_{21} \Gamma_{\mathrm{g}} e^{-j 2 \beta(L-l)}+b_{2}\left(S_{11} \Gamma e^{-j 2 \beta l}-1\right)+b_{3} S_{31} \Gamma_{\mathrm{d}} \\
-S_{31} E_{\mathrm{i}} e^{-j \beta(L-l)}=b_{1} S_{31} \Gamma_{\mathrm{g}} e^{-j 2 \beta(L-l)}+b_{2} S_{31} \Gamma e^{-j 2 \beta l}+b_{3}\left(S_{33} \Gamma_{\mathrm{d}}-1\right) .
\end{array}\right.
$$

Further, we take into account that in real conditions an omnidirectional probe should have little effect on the field in the primary transmission line. This is achieved by a small immersion depth of the probe in the transmission line. Therefore, the modulus of the element of the scattering matrix $S_{31}$ is a small quantity. In addition, we take into account that the modules of the elements of the scattering matrix $S_{11}$ and $S_{33}$, as well as the modules of the CRC from the side of the generator $\Gamma_{\mathrm{g}}$ and the CRC of the quadrature detector $\Gamma_{d}$ are small values (less than 0.1 ), and it is achieved by using ferrite gates, decoupling attenuators, etc. to match. Under these conditions, in the system of equations (3), it is possible without significant loss of accuracy of the model to neglect small quantities of the second and higher orders. Taking it into account, we get:

$$
\left\{\begin{array}{l}
-S_{11} E_{\mathrm{i}} e^{-j \beta(L-l)}=-b_{1}+b_{2} S_{21} \Gamma e^{-j \beta(L-l)} \\
-S_{21} E_{\mathrm{i}} e^{-j \beta(L-l)}=b_{1} S_{21} \Gamma_{\mathrm{g}} e^{-j 2 \beta(L-l)}+b_{2}\left(S_{11} \Gamma e^{-j 2 \beta l}-1\right) \\
-S_{31} E_{\mathrm{i}} e^{-j \beta(L-l)}=b_{2} S_{31} \Gamma e^{-j 2 \beta l}-b_{3} .
\end{array}\right.
$$


Let's solve the system of equations (4) with respect to the branch wave $b_{3}$ incident on the input of the quadrature detector. As a result of the solution, we obtain

$$
b_{3}=E_{\mathrm{i}} S_{31} e^{-\mathrm{j} \beta(L-l)} \frac{1+\left(S_{21}-S_{11}\right) \Gamma e^{-j 2 \beta l}}{1-S_{11} \Gamma e^{-j 2 \beta l}-S_{21}^{2} \Gamma \Gamma_{\mathrm{g}} e^{-j 2 \beta L}} .
$$

It follows from formula (5) that the wave arriving at the input of the quadrature detector from the probe is influenced not only by the CRC of the measured load, but also by the parameters of the probe $\left(S_{11}, S_{21}, S_{31}\right)$, and also by the CRC of the generator $\left(\Gamma_{\mathrm{g}}\right)$, which leads to measurement error. Since the purpose of the article is to study the influence of only an omnidirectional probe on the measurement result, in the formula (5) it is possible to set the $\mathrm{CRC}$ of the generator $\Gamma_{\mathrm{g}}$ to zero, assuming that the generator is ideally aligned with the transmission line. With this in mind, we obtain

$$
b_{3}=E_{\mathrm{i}} e^{-\mathrm{j} \beta(L-l)} S_{31} \frac{1+\left(S_{21}-S_{11}\right) \Gamma e^{-j 2 \beta l}}{1-S_{11} \Gamma e^{-j 2 \beta l}} .
$$

We define the quadrature components $I(l)$ and $Q(l)$ formed at the outputs of the quadrature detector using the relations given, for example, in [11]:

$$
\begin{aligned}
& I(l)=K_{\mathrm{rc}} E_{\mathrm{i}}\left|b_{3}\right| \cdot \cos \left(\psi_{\mathrm{rc}}-\operatorname{argum}\left(b_{3}\right)\right) ; \\
& Q(l)=K_{\mathrm{rc}} E_{\mathrm{i}}\left|b_{3}\right| \cdot \sin \left(\psi_{\mathrm{rc}}-\operatorname{argum}\left(b_{3}\right)\right),
\end{aligned}
$$

$K_{\mathrm{rc}}$ - transmission coefficient of the reference channel; $\left|b_{3}\right|$, $\operatorname{argum}\left(b_{3}\right)-$ modulus and argument of the branched wave $\left|b_{3}\right|$.

We determine the phase distribution of the electromagnetic wave in the transmission line for the case of a non-ideal probe using the well-known formula [11]:

$$
\psi(l)=\operatorname{arctg}(Q(l) / I(l)) \text {. }
$$

Taking into account expressions (7) and (8) for quadrature components, relation (9) takes the following form

$$
\psi(l)=\psi_{\mathrm{rc}}-\operatorname{argum}\left(b_{3}\right) .
$$

Substituting formula (6) in (10), we finally obtain

$$
\psi(l)=\psi_{\mathrm{rc}}+\beta L-\beta l-\varphi_{31}-\operatorname{argum}\left(\frac{1+\left(S_{21}-S_{11}\right) e^{-j 2 \beta l}}{1-S_{11} e^{-j 2 \beta l}}\right),
$$

$\varphi_{31}$ - scatter matrix element argument $S_{31}$.

From formula (11) it follows that the first four components $\left(\psi_{\mathrm{rc}}, \beta L,-\beta l\right.$ and $\left.\psi_{31}\right)$ do not carry information about the measured CRC $\Gamma$, therefore, these components can be excluded when processing the measurement information in the calibration mode of the device. Upon further consideration, we focus on the fifth (informational) component, which we write in the form

$$
\Delta \psi(l)=-\operatorname{argum}\left(\frac{1+\left(S_{21}-S_{11}\right) \Gamma e^{-j 2 \beta l}}{1-S_{11} \Gamma e^{-j 2 \beta l}}\right),
$$


The nature of the influence of the non-ideal probe on the phase distribution of the electromagnetic wave in the transmission line is illustrated in Fig. 2. In this figure, the solid line represents the phase distribution for the non-ideal probe calculated by formula (12), and the dashed line for the ideal probe.

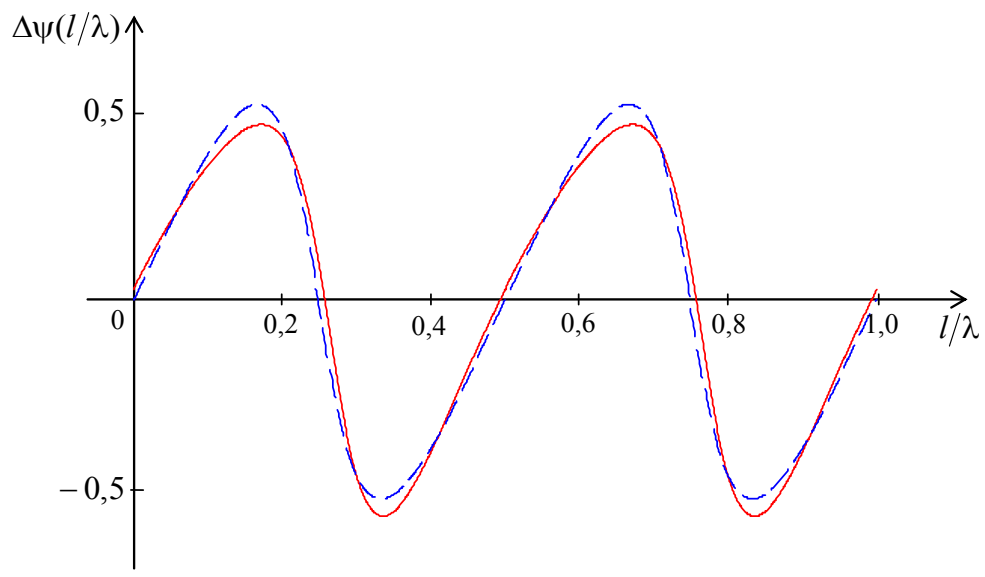

Fig. 2. The nature of the effect of the non-ideal probe on the phase distribution of the electromagnetic wave in the transmission line.

\section{Modelling and calculation of measurement errors}

From the graphs presented it follows that the non-ideal probe leads to the following distortions of the phase distribution:

1. The extreme values of the phase distribution for the case of a non-ideal probe differ from the extreme values for the case of an ideal probe. This leads to an error in measuring the CRC module, which follows from [7, 8] Moreover, the magnitude of the maxima decreases in relation to the ideal situation, and the absolute value of the minima increases. Therefore, it is advisable to use the arithmetic mean value of these quantities in the calculations, which will reduce the measurement error of the CRC module.

2. The zero position of the phase distribution is shifted along the transmission line. This leads to an error in measuring the CRC argument, which follows from [7, 8]. Moreover, the magnitude of the displacement of two adjacent zero values of the phase distribution is different. Therefore, it is necessary to establish which of these values is appropriate to use when measuring the CRC argument from the point of view of minimizing the error.

In order to simulate the errors in the measurement of the module and the argument of the CRC caused by imperfect probe, a program for processing measurement information was created. The program consists of five blocks. In the first block, the initial data are set: the module $|\Gamma|$ and the argument $\varphi$ of the CRC $\Gamma$ of the measured load; modules $\left|S_{21}\right|$, $\left|S_{11}\right|$, and arguments $\varphi_{21}, \varphi_{11}$, of complex $S$ parameters of a non-ideal probe. In the second block, phase distributions are calculated for the case of ideal and non-ideal probes (Fig. 2). The third block automatically searches for two extreme values (maximum and minimum) for the case of a non-ideal probe and two adjacent positions of zero values of phase distributions for the case of ideal and non-ideal probes. In the fourth block, the measured value of the CRC module $\left|\Gamma_{\mathrm{m}}\right|$ and the relative displacement of the zeros of the phase distribution $\Delta l / \lambda(\Delta l-$ absolute displacement) are calculated. When calculating the measured value of the modulus of CRC reflection, we used the formula obtained in [12]: 


$$
\left|\Gamma_{\mathrm{m}}\right|=\sin \left(\Delta \psi_{\max }\right),
$$

$\Delta \psi_{\max }$ - the extreme value of the phase distribution in absolute value (maximum, minimum or average value).

In the fifth block, the relative measurement error of the CRC module is calculated according to the generally accepted formula

$$
\delta \Gamma=\frac{|\Gamma|-\left|\Gamma_{\mathrm{m}}\right|}{|\Gamma|}=1-\frac{\left|\Gamma_{\mathrm{m}}\right|}{|\Gamma|} ;
$$

and the absolute error of measuring the argument according to the formula arising from [12]:

$$
\Delta \varphi=4 \pi \frac{\Delta \mathrm{l}}{\lambda} .
$$

The value of the relative displacement $\Delta l / \lambda$ in the formula (15) can be both positive (zero offset towards the microwave generator) and negative (zero offset towards the measured load). The results of calculations of the relative error in the measurement of the module are presented below in percent ( $\delta \Gamma, \%)$, and the absolute error of the measurement of the argument in degrees ( $\delta \Gamma$, deg.).

To carry out numerical calculations using formulas (12-15), it is necessary to determine the parameters of the elements of the scattering matrix of a non-ideal probe. To this end, electrodynamic modeling was carried out. As a transmission line, a rectangular waveguide with a cross section $23 \times 10 \mathrm{~mm}^{2}$ was chosen. The calculations were carried out for a frequency of $10 \mathrm{GHz}$. The length of the waveguide segment $L$ is chosen equal to the wavelength in the waveguide at this frequency. The probe is made in the form of a metal pin with a diameter of $1 \mathrm{~mm}$ and inserted into the waveguide through a round hole with a diameter of $2 \mathrm{~mm}$, cut in the center of the wide wall. The immersion depth of the probe $h$ into the waveguide segment was varied during electrodynamic modeling. As a result of the simulation, the parameters of the elements of the scattering matrix of the omnidirectional probe at different immersion depths are presented, which are presented in Table 1.

Table 1. Parameters of the elements of the scattering matrix of an omnidirectional probe at various immersion depths.

\begin{tabular}{|c|c|c|c|c|c|c|}
\hline$h, \mathrm{~mm}$ & $\left|S_{31}\right|$ & $\varphi_{31}$, deg. & $\left|S_{21}\right|$ & $\varphi_{21}$, deg. & $\left|S_{11}\right|$ & $\varphi_{11}$, deg. \\
\hline 1 & 0.005 & 88.18 & 1.000 & -0.10 & 0.001 & -89.71 \\
\hline 2 & 0.017 & 86.58 & 1.000 & -0.26 & 0.002 & -92.19 \\
\hline 3 & 0.040 & 84.17 & 0.999 & -0.72 & 0.009 & -95.91 \\
\hline 4 & 0.087 & 79.96 & 0.996 & -1.91 & 0.003 & -99.31 \\
\hline 5 & 0.192 & 70.87 & 0.978 & -4.97 & 0.085 & -107.85 \\
\hline
\end{tabular}

Using the results presented in Table 1 according to formulas (12-15) using the developed program, the calculation of measurement errors caused by imperfect probe was carried out at various depths of immersion. The calculations were performed for the load with the following parameters: $|\Gamma|=0.5, \varphi=0$. The results are presented in Table 2 . 
Table 2. Calculations of measurement errors caused by imperfect probe at various depths of immersion.

\begin{tabular}{|c|c|c|c|c|c|c|}
\hline$h, \mathrm{~mm}$ & $\begin{array}{c}\delta \Gamma, \% \\
\text { to the } \\
\text { maximum }\end{array}$ & $\begin{array}{c}\delta \Gamma, \% \\
\text { to a } \\
\text { minimum }\end{array}$ & $\begin{array}{c}\delta \Gamma, \% \\
\text { averaging }\end{array}$ & $\begin{array}{c}\Delta \varphi, \text { deg. } \\
\text { first zero }\end{array}$ & $\begin{array}{c}\Delta \varphi, \text { deg. } \\
\text { second } \\
\text { zero }\end{array}$ & $\begin{array}{c}\Delta \varphi, \text { deg. } \\
\text { averaging }\end{array}$ \\
\hline 1 & 0.04 & -0.04 & 0.00 & 0.07 & 0.13 & 0.10 \\
\hline 2 & 0.09 & -0.09 & 0.00 & 0.20 & 0.32 & 0.26 \\
\hline 3 & 0.47 & -0.31 & 0.08 & 0.46 & 0.97 & 0.72 \\
\hline 4 & 1.57 & -1.00 & 0.28 & 1.06 & 2.76 & 1.91 \\
\hline 5 & 5.16 & -1.94 & 1.59 & 2.60 & 7.34 & 4.97 \\
\hline
\end{tabular}

The data presented in Table 2 allow us to draw the following conclusions. With an increase in the immersion depth of the probe into the waveguide, the errors in the measurements of the module and the CRC argument increase. Moreover, when calculating the CRC module using the minimum phase distribution, the error is 1.5 times less than when using the maximum. The expediency of using averaging of extreme values in the calculation of the CRC module is confirmed, which leads to an additional increase in accuracy. It was established that to calculate the CRC argument, it is advisable to use the zero phase distribution, in the region of which this distribution has a higher slope (in Fig. 2 this is the first zero on the generator side). The use of averaging of the displacements of the first and second zeros of the phase distribution when measuring the CRC argument is impractical, since as a result of averaging, the resulting error significantly exceeds the error when using the first zero.

In practical implementation, probes are used that are weakly coupled to the field in the transmission line with a transitional attenuation of at least $20 \mathrm{~dB}\left(\left|S_{31}\right| \leq 0.1\right)$. In our case, this corresponds to a probe immersion depth of $h \leq 4 \mathrm{~mm}$ (see Table 1). Moreover, from Table 2 it follows that the measurement error of the module when using the minimum phase distribution does not exceed $1 \%$ (when averaging - not more than $0.28 \%$ ), and the error in measuring the argument when using the first zero of the phase distribution does not exceed 1.06 degrees.

Fig. 3 presents graphs of the dependence of the measurement errors of the module $(a)$ and argument $(b)$ of the CRC on the value of the module. The calculations were performed for two values of the immersion depth of the probe $h$, equal to $3 \mathrm{~mm}$ and $4 \mathrm{~mm}$. In the calculations, the averaging of extreme values was used to determine the CRC modulus and the first zero of the phase distribution to determine the CRC argument.

From the presented dependencies, it follows that the measurement errors of the module and the $\mathrm{CRC}$ argument, caused by the imperfection of the probe when using the phase distribution of the field in the transmission line, increase with decreasing module. Moreover, for practically important cases $(h \leq 4 \mathrm{~mm})$, in the range of variation of the CRC module from 0.05 to 1 , the relative error in the measurement of the module does not exceed $0.5 \%$, and the absolute error in the measurement of the argument does not exceed 2 degrees.

Using the developed program, we also studied the dependence of the errors in the measurement of the module and the CRC argument on the argument value. The calculations showed that these errors are practically independent of the value of the $\mathrm{CRC}$ argument.

\section{Conclusion}

We performed a study of the influence of non-ideal non-directional probe on the error of the quadrature method for measuring the modulus and argument of the CRC of microwave 
nodes. As a result, it was shown that the additional errors caused by the influence of the probe on the structure of the phase distribution of the electromagnetic wave in the transmission line do not exceed $0.5 \%$ in absolute value and 2 degrees in argument for practically important cases. This allows us to conclude that it is possible to create, on the basis of the quadrature method of measurement, high-precision and inexpensive measuring transducers (sensors) that can be used for the purpose of the built-in control of the parameters of microwave paths of electronic systems and for the purpose of microwave control of the parameters of technological processes.

a)
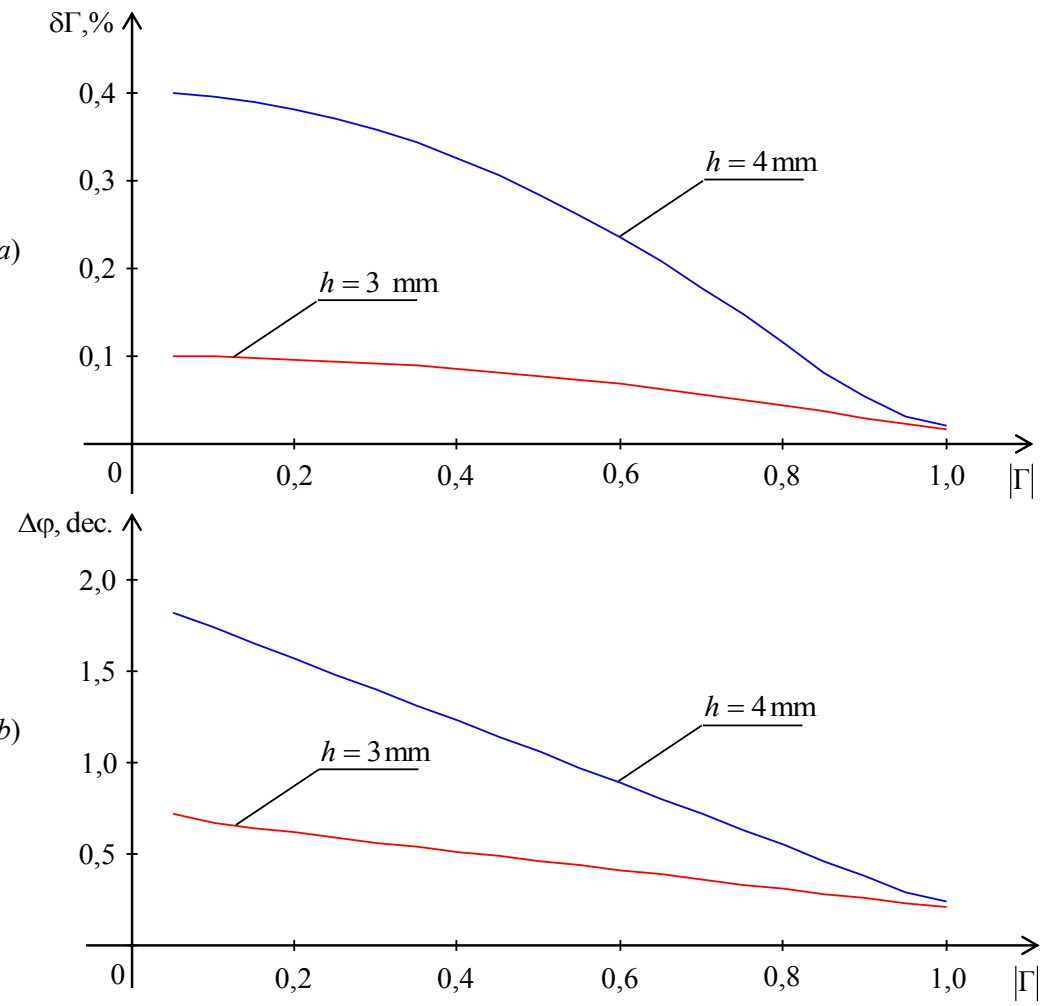

Fig. 3. Dependences of the measurement errors of the module and the CRC argument on the value of the module.

\section{References}

1. A.A. Danilin, Izmereniya v tehnike SVCh (Radiotehnika, 2008)

2. B.V. Dvorashkin, Osnovy metrologii i radioizmereniya (Radio i svyaz', 1993)

3. I.K. Bondarenko, G.A. Dejnega, Z.V. Magrachev, Avtomatizaciya izmerenij parametrov SVCh traktov (Sov. radio, 1969)

4. B.A. Abubakirov, K.P. Gudkov, E.V. Nechaev, Izmerenie parametrov radiotehnicheskih cepej (Radio i svyaz, 1984)

5. Y.B. Gimpilevich, Y.Y. Smailov, A method for measuring of two microwave signals vector ratio, Proceedings of the 5th International Conference on Antenna Theory and Techniques (ICATT), Kyiv, pp. 397-398 (2005)

6. Gimpilevich Yu.B, Sposob opredeleniya modulya i fazy koefficienta otrazheniya SVChdvupolyusnika. Patent USSR, no. 4407549 (1991) 
7. Y.B. Gimpilevich, S.E. Zebek, Quadrature measurement method for complex parameters of microwave two-poles, Journal of the Russian universities: Radioelectronics., № 1. — pp. 29 - 38 (2019)

8. Gimpilevich Yu.B, Zebek S.E. Sposob opredeleniya modulya i argumenta kompleksnogo koefficienta otrazheniya mikrovolnovogo dvuhpolyusnika. Patent RF, no. 2683804 (2019)

9. Gimpilevich Yu.B, Zebek S.E. Ustrojstvo dlya izmereniya modulya i argumenta kompleksnogo koefficienta otrazheniya mikrovolnovogo dvuhpolyusnika. Patent RF, no. 189436 (2019)

10. M. A. Silaev, S.F. Bryancev, Prilozhenie matric i grafov k analizu SVCh ustrojstv (Sov. Radio, 1970)

11. Richard G. Lyons Understanding Digital Signal Processing, 3rd Edition, (Prentice Hall, 2011)

12. Y.B. Gimpilevich, Izmerenie i kontrol parametrov mikrovolnovyh traktov (SevNTU, 2009) 